\title{
Double stratification effects on heat and mass transfer in unsteady MHD nanofluid flow over a flat surface
}

\author{
Winifred Nduku Mutuku ${ }^{1 *}$ and Oluwole Daniel Makinde ${ }^{2}$
}

${ }^{*}$ Correspondence:
mutukuwinnie@gmail.com
${ }^{1}$ Department
of Mathematics, Kenyatta
University, Nairobi, Kenya
Full list of author information
is available at the end of the
article

*Correspondence: mutukuwinnie@gmail.com of Mathematics, Kenyatta University, Nairobi, Kenya

\begin{abstract}
The focus of this work is to theoretically investigate the problem of double stratification on heat and mass transfer in an unsteady hydromagnetic boundary layer flow of a nanofluid over a flat surface. The model employed for the nanofluid transport equations incorporate the effects of Brownian motion and thermophoresis in the presence of thermal and solutal stratification. The governing nonlinear partial differential equations and their associated boundary conditions are initially transformed into dimensionless form by using similarity variables, before being solved numerically by employing the Runge-Kutta-Fehlberg fourth-order method with shooting technique. The effects of different controlling parameters, viz. solutal and thermal stratification, Lewis number, thermophoresis, Brownian motion, magnetic field and unsteadiness on the fluid velocity, temperature, skin friction coefficient, the local Nusselt number, and the local Sherwood number are graphically depicted and quantitatively discussed in detail taking into account the practical applications of each profile. It is noted that thermal stratification reduces the fluid temperature, while the solutal stratification reduces the nanoparticle concentration.
\end{abstract}

Keywords: Heat transfer, Mass transfer, Unsteady, MHD, Nanofluid, Double stratification

\section{Background}

Magnetohydrodynamics (MHD) boundary layer flow of electrically conducting fluids has diverse industrial and engineering applications in fields such as nuclear reactors, geothermal engineering, liquid metals and plasma flows, petroleum industries, boundary layer control in aerodynamics and crystal growth. Several authors have studied the problem of MHD boundary layer flow, heat and mass transfer about different surface geometries in electrically conducting fluids. Makinde [1] numerically analysed the influence of magnetic field on the steady heat and mass flow of an electrically conducting fluid by mixed convection along a semi-infinite vertical porous plate with constant heat flux taking into account Soret and Dufour effects. The effect of the Hall current on the MHD natural convection flow from a vertical permeable flat plate with a uniform heat flux in the presence of a transverse magnetic field was analysed by Saha et al. [2]. Rout et al. [3] investigated MHD heat and mass transfer of chemically reacting fluid flow over a moving vertical plate in the presence of a heat source with convective boundary condition.

(c) The Author(s) 2017. This article is distributed under the terms of the Creative Commons Attribution 4.0 International License (http://creativecommons.org/licenses/by/4.0/), which permits unrestricted use, distribution, and reproduction in any medium, provided you give appropriate credit to the original author(s) and the source, provide a link to the Creative Commons license, and indicate if changes were made. 
Stratification is the layering of a fluid system due to temperature differences and variations in concentration or the presence of different fluids with varying densities. In practical situations where the heat and mass transfer mechanisms occur simultaneously, it is important to analyse the effect of double stratification (stratification of medium with respect to thermal and concentration fields) on the convective transport in the fluid. The effect of double stratification is important in applications involving fluids' convective transport where heat and mass transfer run concurrently. It is encountered in several classical problems, for example, reservoir mixing, internal waves, shear flow instability, internal hydraulics, turbulence and jets, plumes and wakes. Stratified fluids are omnipresent in nature and their occurrence is very common in heterogeneous fluid bodies, such as thermal stratification of reservoirs and oceans, salinity stratification in estuaries, rivers, groundwater reservoirs, and oceans, heterogeneous mixtures in industrial food manufacturing processing, density stratification of the atmosphere, and uncountable similar examples [4]. For instance, density variation causes thermal stratification in reservoirs which leads to a reduction in the vertical mixing of oxygen to the point that bottom water becomes anoxic through the action of biological processes. Preventing, predicting, and solving such a reservoir problem, though dependent on other limnological factors, require an understanding of the dynamics of stratified fluids. MHD-convective heat transfer in thermally stratified fluid occurs in many industrial applications and is an important aspect in the study of heat transfer. Higher energy efficiency and increased system performance can be achieved with better thermal stratification. This has led to intensified research in mass and heat transfer in thermally stratified medium [5-7]. However, the aforementioned investigations analysed natural convection flow of convectional heat transfer fluids.

Recently with the advent of nanofluids - a term coined by Choi [8] referring to a liquid containing a suspension of nanometre-sized solid particle (nanoparticles) - research has diversified to convective heat and mass transfer of nanofluids in doubly stratified medium. Although the effect of double stratification on heat and mass transfer in a fluid is significant, very little work has been reported in the literature [9-11]. On the other hand, studies on unsteady boundary layer flow, mass transfer, and convective heat transfer in a nanofluid are the topics of contemporary research areas in fluid science and engineering, owing to its novelty [12-17]. The mass and heat transfer in a doubly stratified medium in the presence of a magnetic field is not only fundamentally interesting but also finds wide range of natural, industrial, and engineering applications. These applications include heat rejection into the environment such as lakes, rivers, and seas; thermal energy storage systems such as solar ponds; heat transfer from thermal sources such as condensers of power plants.

Despite its numerous applications, it is evident from the literature herein that research has neglected mass and heat transfer in unsteady MHD nanofluid flow taking into account thermophoresis and Brownian motion in a doubly stratified medium. Hence, the motivation of this paper is to bridge this knowledge gap. This study aims to extend the recent work of Olanrewaju and Makinde [18] to incorporate unsteadiness, magnetic field, and the effects of double stratification on boundary layer nanofluid flow. The shooting iteration technique coupled with fourth-order Runge-Kutta integration scheme is employed to numerically solve the model nonlinear system of equations of 
this particular problem. Numerical results are displayed graphically and discussed for different values of the various dimensionless parameters controlling the flow regime. It is hoped that the results obtained will not only provide useful information for applications but also serve as a basis for studying other analogous systems that arise in engineering and industrial applications.

\section{Model formulation}

We consider heat and mass transfer in an unsteady flow of an incompressible electrically conducting viscous nanofluid past an infinite nonconducting flat plate in the presence of a transversely imposed unsteady magnetic field of strength $B(t)$. The sketch of the physical configuration and coordinate system is shown in Fig. 1. The induced magnetic field is neglected, which is justified for industrial application MHD flows at small magnetic Reynolds number. It is also assumed that the external electrical field is zero and that the electric field due to the polarization of charges is also negligible. The temperature and nanoparticle concentration at the plate surface and at the free stream are assumed to vary with time and axial distance along the plate.

Under the above assumptions with the boundary layer approximations, the continuity, momentum, energy, and nanoparticle concentration equations are as follows:

$$
\begin{aligned}
\frac{\partial u}{\partial x}+\frac{\partial v}{\partial y}=0, & \\
\frac{\partial u}{\partial t}+u \frac{\partial u}{\partial x}+v \frac{\partial u}{\partial y}= & \frac{\partial U_{\infty}}{\partial t}+U_{\infty} \frac{\partial U_{\infty}}{\partial x}+v \frac{\partial^{2} u}{\partial y^{2}}-\frac{\sigma B(t)^{2}\left(u-U_{\infty}\right)}{\rho} \\
\frac{\partial T}{\partial t}+u \frac{\partial T}{\partial x}+v \frac{\partial T}{\partial y}= & \frac{\partial T_{\infty}}{\partial t}+U_{\infty} \frac{\partial T_{\infty}}{\partial x}+\alpha \frac{\partial^{2} T}{\partial y^{2}}+\frac{v}{c_{\mathrm{p}}}\left(\frac{\partial u}{\partial y}\right)^{2} \\
& -\frac{\sigma B(t)^{2}\left(u-U_{\infty}\right)^{2}}{\rho c_{\mathrm{p}}}+\tau\left\{D_{\mathrm{B}} \frac{\partial C}{\partial y} \frac{\partial T}{\partial y}+\frac{D_{T}}{T_{0, \infty}}\left(\frac{\partial T}{\partial y}\right)^{2}\right\}
\end{aligned}
$$

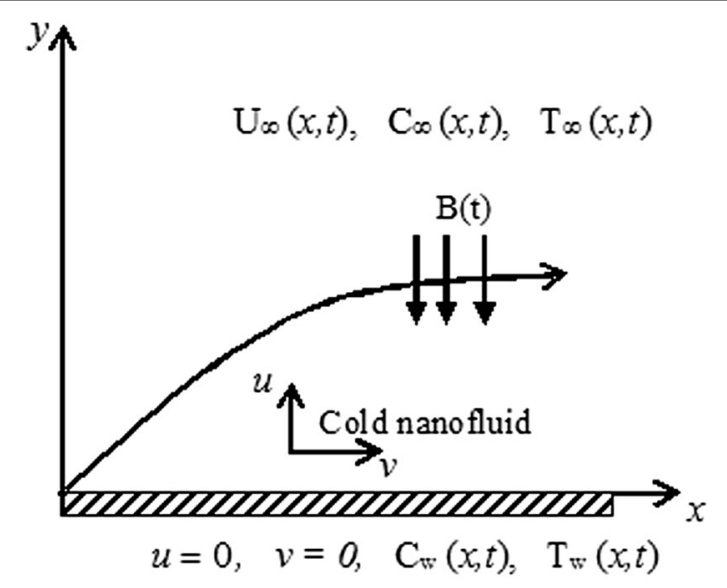

Fig. 1 Physical configuration 


$$
\frac{\partial C}{\partial t}+u \frac{\partial C}{\partial x}+v \frac{\partial C}{\partial y}=\frac{\partial C_{\infty}}{\partial t}+U_{\infty} \frac{\partial C_{\infty}}{\partial x}+D_{\mathrm{B}} \frac{\partial^{2} C}{\partial y^{2}}+\frac{D_{\mathrm{T}}}{T_{0, \infty}} \frac{\partial^{2} T}{\partial y^{2}}
$$

with the boundary conditions

$$
\begin{aligned}
& u=0, v=0, T=T_{\mathrm{w}}(x, t), C=C_{\mathrm{w}}(x, t) \quad \text { at } y=0, \\
& u \rightarrow U_{\infty}(x, t), C \rightarrow C_{\infty}(x, t), T \rightarrow T_{\infty}(x, t), \quad \text { as } y \rightarrow \infty,
\end{aligned}
$$

where $u$ and $v$ are the velocity components along the $x$ and $y$ directions, respectively; $t$ is time; $T_{\mathrm{w}}$ and $C_{\mathrm{w}}$ are temperature and nanoparticle volume fraction at the plate surface, respectively; $U_{\infty}, T_{\infty}$, and $C_{\infty}$ are the free stream velocity, temperature, and nanoparticle volume fraction, respectively; $\rho$ is the density; $\alpha$ is the thermal diffusivity; $v$ is the kinematic viscosity; $c_{\mathrm{p}}$ is the specific heat at constant pressure; $\sigma$ is the electrical conductivity; $\tau=(\rho C)_{\mathrm{p}} /(\rho C)_{\mathrm{f}}$ is the ratio of the effective heat capacitance of the nanoparticle to that of the base fluid; $D_{\mathrm{B}}$ is the Brownian motion coefficient; $D_{\mathrm{T}}$ is the thermophoretic coefficient; $T_{0, \infty}$ is the constant free stream temperature; and $C$ is the nanoparticle volume fraction.

Following Ibrahim and Shankar [19], we prescribe the free stream velocity and the stratified free stream and plate surface temperature and nanoparticles concentration together with the unsteady magnetic field as follows:

$$
\begin{gathered}
U_{\infty}(x, t)=\frac{a x}{1-\lambda t}, T_{\mathrm{w}}(x, t)=T_{0, \infty}+\frac{b x}{1-\lambda t}, T_{\infty}(x, t)=T_{0, \infty}+\frac{c x}{1-\lambda t}, \\
B(t)=\frac{B_{0}}{\sqrt{1-\lambda t}}, C_{\mathrm{w}}(x, t)=C_{0, \infty}+\frac{m x}{1-\lambda t}, C_{\infty}(x, t)=C_{0, \infty}+\frac{n x}{1-\lambda t},
\end{gathered}
$$

where $\lambda t<1, a, b, c, m$, and $n$ are positive constants and $\lambda$ with dimension (time) $)^{-1}$ is unsteadiness frequency parameter. Positive values of $b, c, n$, and $m$ correspond to the assisting flows and their negative values correspond to opposing flows. In order to simplify the mathematical analysis of the problem, we introduce the following dimensionless variables:

$$
\begin{aligned}
& \eta=\sqrt{\frac{a}{v(1-\lambda t)}} y, \psi=\sqrt{\frac{a v}{(1-\lambda t)}} x f(\eta), \\
& \theta(\eta)=\frac{T-T_{\infty}}{T_{\mathrm{w}}-T_{0, \infty}}=\frac{T-T_{0, \infty}}{T_{\mathrm{w}}-T_{0, \infty}}-\frac{c x}{(1-\lambda t)\left(T_{\mathrm{w}}-T_{0, \infty}\right)}, \\
& \phi(\eta)=\frac{C-C_{\infty}}{C_{\mathrm{w}}-C_{0, \infty}}=\frac{C-C_{\infty}}{C_{\mathrm{w}}-C_{0, \infty}}-\frac{n x}{(1-\lambda t)\left(C_{\mathrm{w}}-C_{0, \infty}\right)},
\end{aligned}
$$

where $\eta$ is the similarity variable and $\psi$ is the stream function defined as

$$
u=\frac{\partial \psi}{\partial y} \quad \text { and } \quad v=-\frac{\partial \psi}{\partial x}
$$

Substituting Eqs. (7) and (8) into Eqs. (1) and (4), we obtain following system of similarity equations:

$$
f^{\prime \prime \prime}+f^{\prime \prime}-f^{\prime 2}-A\left(f^{\prime}+\frac{\eta}{2} f^{\prime \prime}-1\right)-H a\left(f^{\prime}-1\right)+1=0
$$




$$
\begin{aligned}
& \theta^{\prime \prime}-A \operatorname{Pr}\left(\frac{\eta \theta^{\prime}}{2}+\theta\right)+\operatorname{Pr}\left(f \theta^{\prime}+\beta_{1}-f^{\prime} \theta-f^{\prime} \beta_{1}\right) \\
& \quad+\operatorname{Pr} E c\left[\left(f^{\prime \prime}\right)^{2}+H a\left(f^{\prime}-1\right)^{2}\right]+N_{\mathrm{b}} \theta^{\prime} \phi^{\prime}+N_{\mathrm{t}}\left(\theta^{\prime}\right)^{2}=0 \\
& \phi^{\prime \prime}-A L e\left(\frac{\eta \phi^{\prime}}{2}+\phi\right)+\operatorname{Le}\left(f \phi^{\prime}+\beta_{2}-f^{\prime} \phi-f^{\prime} \beta_{2}\right)+\frac{N_{\mathrm{t}}}{N_{\mathrm{b}}} \theta^{\prime \prime}=0,
\end{aligned}
$$

subject to the boundary conditions

$$
\begin{aligned}
& f(0)=0, f^{\prime}(0)=0, \theta(0)=1-\beta_{1}, \phi(0)=1-\beta_{2}, \\
& f^{\prime}(\infty)=1, \theta(\infty)=0, \phi(\infty)=0,
\end{aligned}
$$

where primes denote differentiation with respect to $\eta$ and $H a$ is Hartmann number, $P r$ is Prandtl number, $N_{\mathrm{b}}$ is the Brownian motion parameter, $N_{\mathrm{t}}$ is the thermophoresis parameter, $E c$ is Eckert number, $L e$ is Lewis number, $A$ is the unsteadiness parameter, $\beta_{1}$ is the thermal stratification parameter, and $\beta_{2}$ is the solutal stratification parameter.

The above parameters are defined as follows:

$$
\begin{aligned}
& H a=\frac{\sigma B_{0}^{2}}{a \rho}, \operatorname{Pr}=\frac{v}{\alpha}, N_{\mathrm{b}}=\frac{\tau D_{\mathrm{B}}\left(C_{\mathrm{w}}-C_{0, \infty}\right)}{v}, N_{\mathrm{t}}=\frac{\tau D_{\mathrm{T}}\left(T_{\mathrm{w}}-T_{0, \infty}\right)}{T_{o, \infty} v}, \\
& E c=\frac{U_{\infty}^{2}}{C_{\mathrm{p}}\left(T_{\mathrm{w}}-T_{0, \infty}\right)}, L e=\frac{v}{D_{\mathrm{m}}}, A=\frac{\lambda}{a}, \beta_{1}=\frac{c}{b}, \beta_{2}=\frac{n}{m} .
\end{aligned}
$$

The significant physical quantities of engineering and industrial interest in this study are the skin friction coefficient $C_{\mathrm{f}}$, Nusselt number $\mathrm{Nu}$, and Sherwood number $\mathrm{Sh}$ defined, respectively, as

$$
C_{\mathrm{f}}=\frac{\tau_{w}}{\rho u_{\infty}^{2}}, N u=\frac{x q_{\mathrm{w}}}{k\left(T_{\mathrm{w}}-T_{0, \infty}\right)}, S h=\frac{x q_{\mathrm{m}}}{D_{\mathrm{B}}\left(C_{\mathrm{w}}-C_{0, \infty}\right)},
$$

where $\tau_{\mathrm{w}}, q_{\mathrm{w}}$, and $q_{\mathrm{m}}$ are the skin friction, surface heat flux, and the surface mass flux, respectively, defined by

$$
\tau_{\mathrm{w}}=\left.\mu \frac{\partial u}{\partial y}\right|_{y=0}, q_{\mathrm{w}}=-\left.k \frac{\partial T}{\partial y}\right|_{y=0}, q_{\mathrm{m}}=-\left.D_{\mathrm{B}} \frac{\partial C}{\partial y}\right|_{y=0} .
$$

where $\mu$ and $k$ are the dynamic viscosity and the thermal conductivity, respectively.

Using Eqs. (15) into (14), we get

$$
R e_{x}^{1 / 2} C_{\mathrm{f}}=f^{\prime \prime}(0), R e_{x}^{-1 / 2} N u=-\theta^{\prime}(0), R e_{x}^{-1 / 2} S h=-\phi^{\prime}(0),
$$

which are the local skin friction $C_{\mathrm{f}}$, local Nusselt number $\mathrm{Nu}$, and local Sherwood number $S h$, respectively, and $R e_{x}=U_{\infty} x / v$ is the local Reynolds number. 


\section{Numerical procedure}

The system of nonlinear differential Eqs. (9)-(11) with the boundary conditions (12) has been solved numerically using shooting technique coupled with the fourth-order Runge-Kutta method and a modified version of the Newton-Raphson algorithm.

Let

$$
x_{1}=f, x_{2}=f^{\prime}, x_{3}=f^{\prime \prime}, x_{4}=\theta, x_{5}=\theta^{\prime}, x_{6}=\phi, x_{7}=\phi^{\prime} .
$$

Then the set of higher order boundary value equations with their respective boundary conditions is reduced to a system of first-order differential equation with appropriate initial conditions, respectively,

$$
\begin{aligned}
x_{1}^{\prime}= & x_{2}, \\
x_{2}^{\prime}= & x_{3}, \\
x_{3}^{\prime}= & -x_{1} x_{3}+x_{2}^{2}+A\left(x_{2}+\frac{\eta}{2} x^{3}-1\right)+H a\left(x_{2}-1\right)-1, \\
x_{4}^{\prime}= & x_{5}, \\
x_{5}^{\prime}= & A \operatorname{Pr}\left(\frac{\eta x_{5}}{2}+x_{4}\right)-\operatorname{Pr}\left(x_{1} x_{5}+\beta_{1}-x_{2} x_{4}-x_{2} \beta_{1}\right) \\
& -\operatorname{Pr} E c\left[x_{3}^{\prime}+H a\left(x_{2}^{\prime}-1\right)\right]-N_{\mathrm{b}} x_{5} x_{7}-N_{\mathrm{t}} x_{5}^{2}, \\
x_{6}^{\prime}= & x_{7}, \\
x_{7}^{\prime}= & A \operatorname{Le}\left(\frac{\eta x_{7}}{2}+x_{6}\right)+\operatorname{Le}\left(x_{1} x_{7}+\beta_{2}-x_{2} x_{6}-x_{2} \beta_{2}\right) \\
& -\frac{N_{\mathrm{t}}}{N_{\mathrm{b}}}\left\{\operatorname{APr}\left(\frac{\eta x_{5}}{2}+x_{4}\right)-\operatorname{Pr}\left(x_{1} x_{5}+\beta_{1}-x_{2} x_{4}-x_{2} \beta_{1}\right)\right\},
\end{aligned}
$$

subject to the initial conditions

$$
\begin{aligned}
& x_{1}(0)=0, x_{2}(0)=0, x_{3}(0)=s_{1}, x_{4}(0)=1-\beta_{1}, \\
& x_{5}(0)=s_{2}, x_{6}(0)=1-\beta_{2}, x_{7}=s_{3},
\end{aligned}
$$

By applying the shooting method, the unspecified initial conditions $S_{1}, S_{2}$, and $S_{3}$ in (19) are assumed and (18) integrated numerically as an initial valued problem to a given terminal point. The accuracy of the assumed missing initial conditions was checked by comparing the calculated value of the dependent variable at the terminal point with its given value there. If differences exist, improved values of the missing initial conditions are obtained and the process repeated. The method is programmed in MAPLE with the step size of $\Delta \eta=0.001$ selected to be satisfactory for a convergence criterion of $10^{-7}$ in nearly all cases. The maximum value of $\eta_{\infty}$ to each group of parameters was determined when the values of the unknown boundary conditions at $\eta=0$ do not change to successful loop with error less than $10^{-7}$.

\section{Results and discussion}

From the numerical computation process, the effects of the pertinent parameters, namely, unsteadiness parameter $A$, Lewis number $L e$, Brownian parameter $N_{\mathrm{b}}$, thermophoresis parameter $N_{\mathrm{t}}$, Hartmann number $H a$, Eckert number $E c$, thermal stratification parameter $\beta_{1}$, and solutal stratification parameter $\beta_{2}$ on the fluid velocity, temperature, nanoparticles volume as well as the skin friction coefficient, the local 
Nusselt number, and the local Sherwood number are illustrated graphically (Figs. 2, 3, $4,5,6,7,8,9,10,11,12,13,14)$ and analysed quantitatively highlighting their industrial and engineering applications. In order to ascertain the validity of our numerical procedure, the special case of heat transfer in flow of a convectional fluid in the absence of buoyancy force, flow unsteadiness, magnetic field, and double stratification effect (i.e.

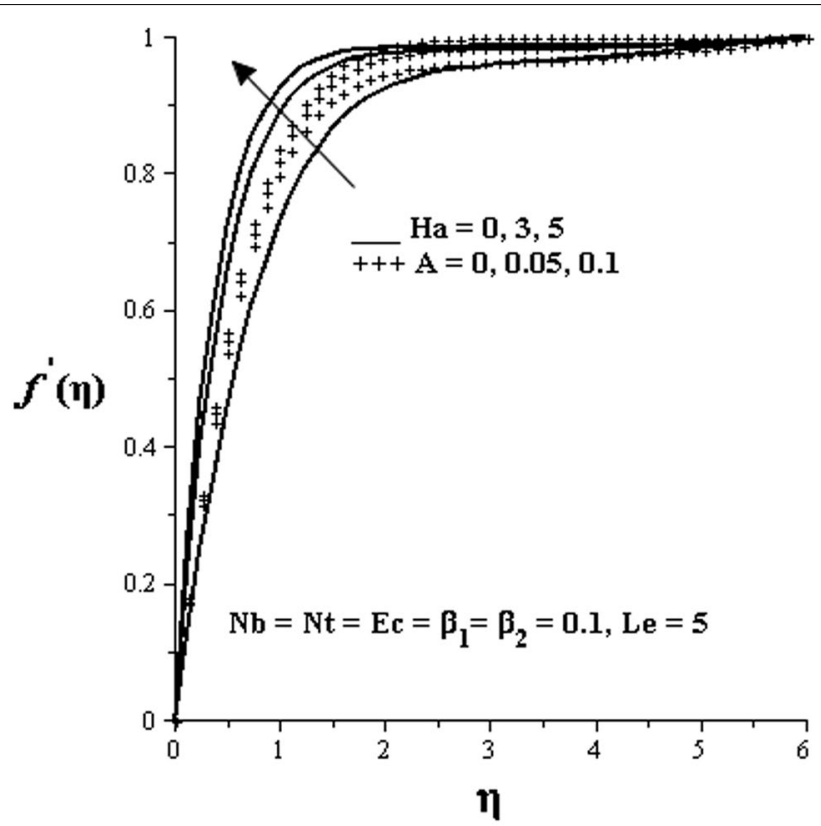

Fig. 2 Effects of $A$ and $\mathrm{Ha}$ on dimensionless velocity

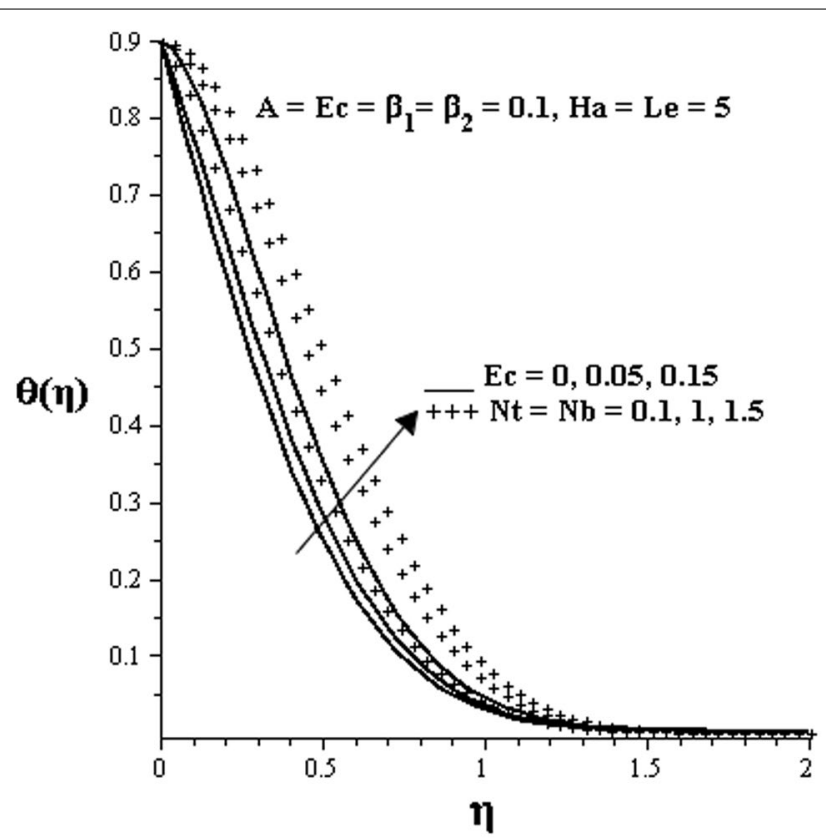

Fig. 3 Effects of $E c, N t$, and $\mathrm{Nb}$ on dimensionless temperature 


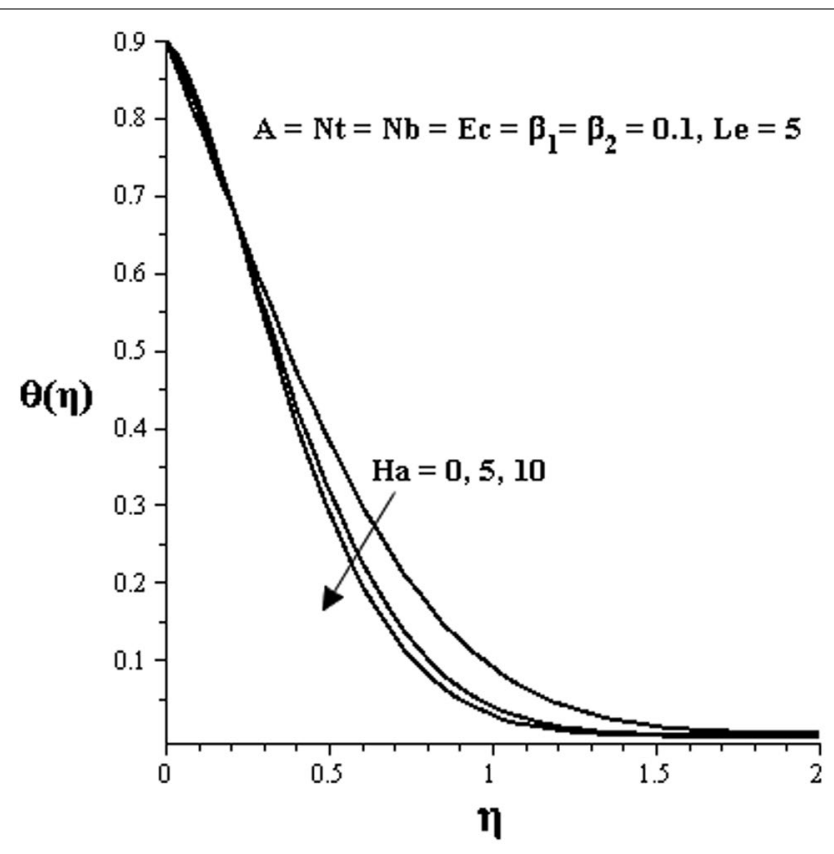

Fig. 4 Effects of $\mathrm{Ha}$ on dimensionless temperature

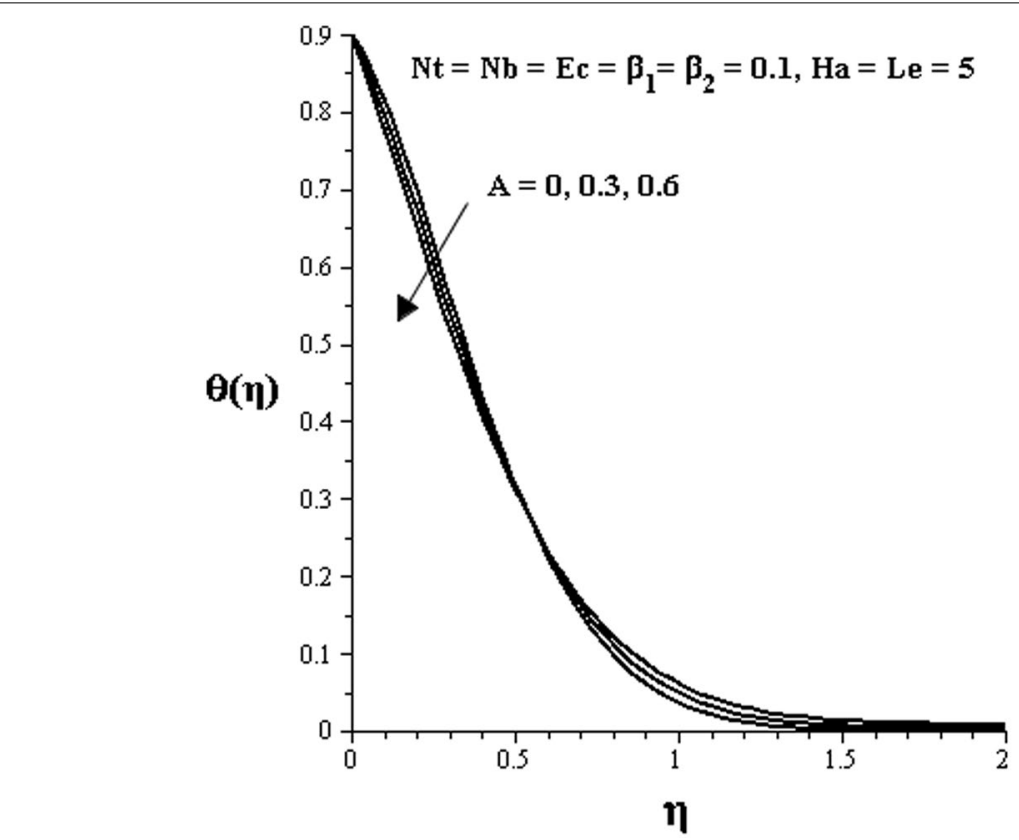

Fig. 5 Effects of $A$ on dimensionless temperature

$\mathrm{A}=\beta_{1}=\beta_{2}=H a=\phi=0$ ) over a surface can be compared with that of Aman et al. [20], as shown in Table 1 and excellent agreement was established. This favourable comparison lends confidence to the numerical results reported subsequently. 


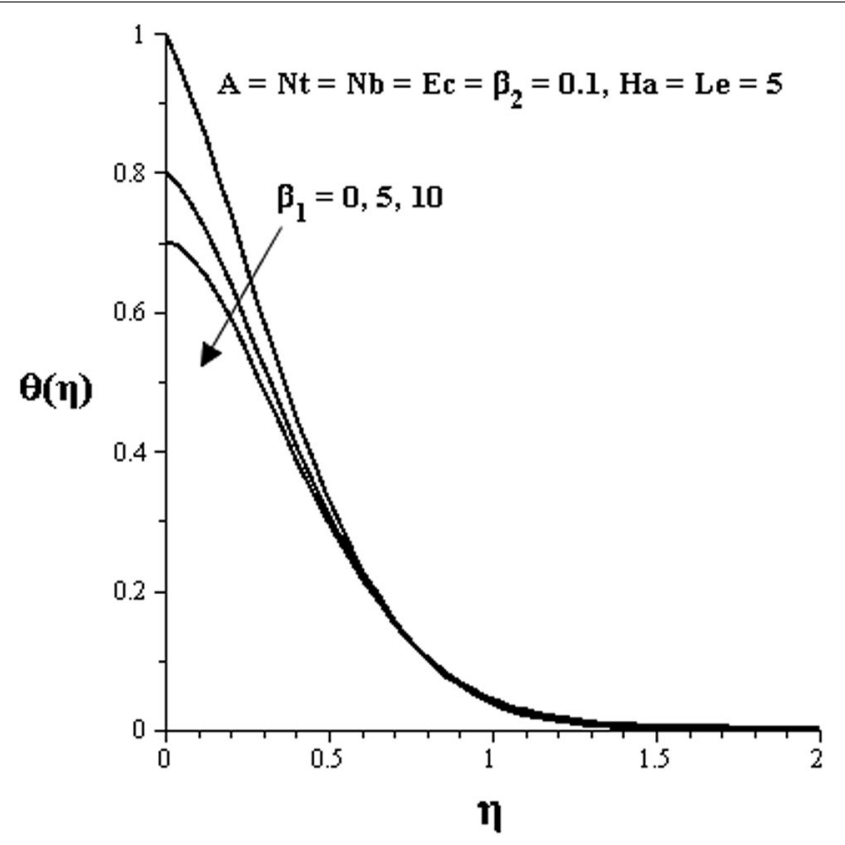

Fig. 6 Effects of $\beta_{1}$ on dimensionless temperature

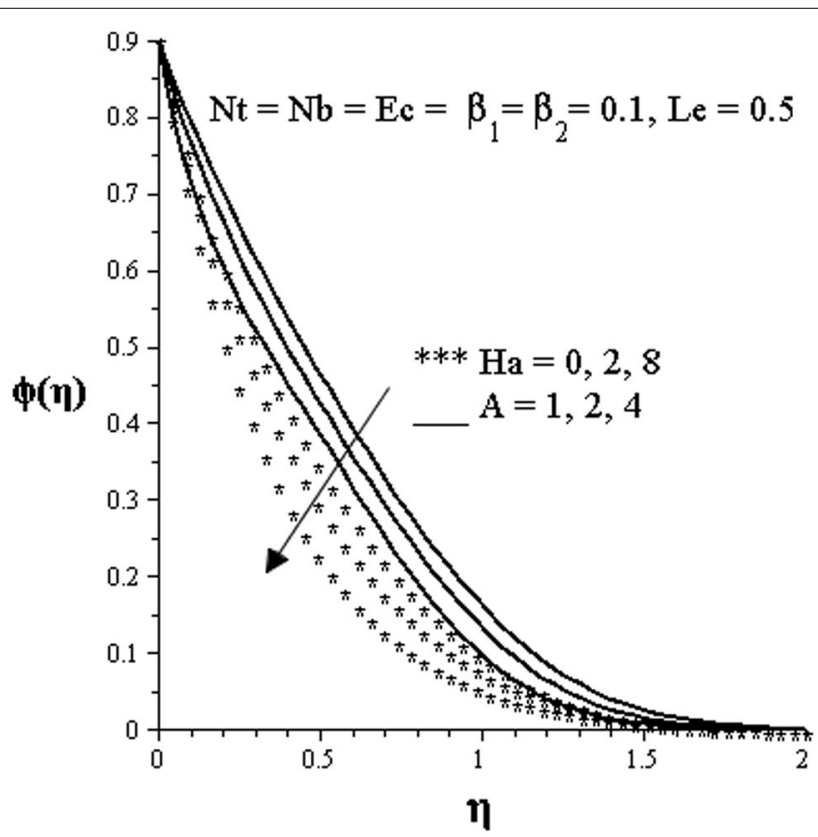

Fig. 7 Effects of $A$ and $\mathrm{Ha}$ on dimensionless nanoparticle concentration

\section{Velocity profiles}

Figure 2 shows the effect of unsteadiness and magnetic field strength on the nanofluids' velocity profiles. It can be seen that there is a gradual increase in the fluid velocity, from zero at the plate surface to a maximum value as it approaches the free stream far away from the plate surface thus satisfying the boundary conditions. It is observed 


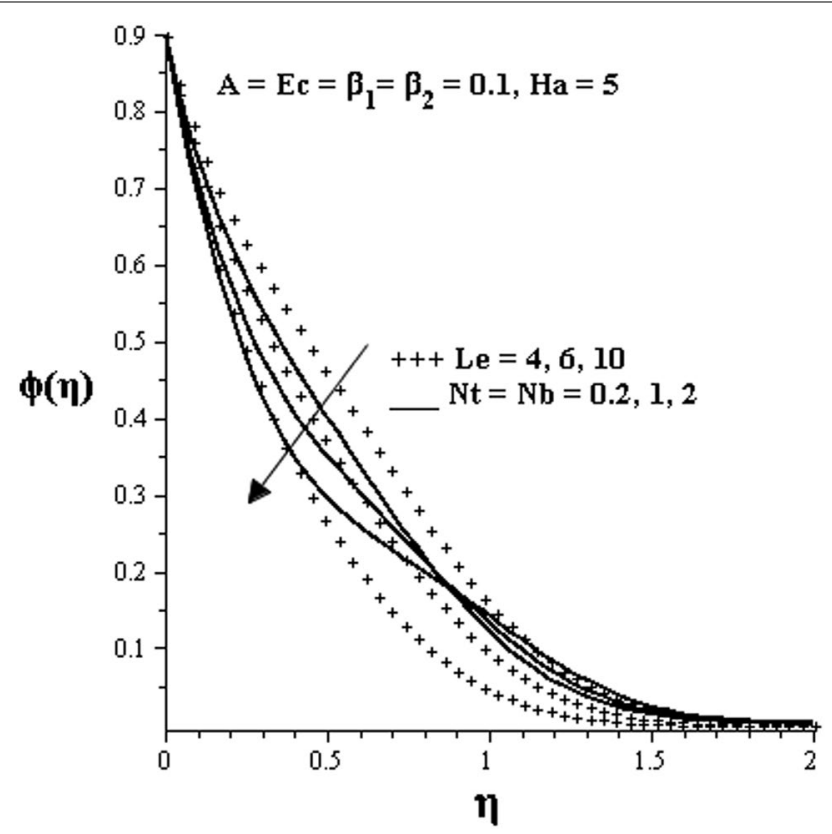

Fig. 8 Effects of $L e, N \mathrm{Nt}$, and $\mathrm{Nb}$ on dimensionless nanoparticle concentration

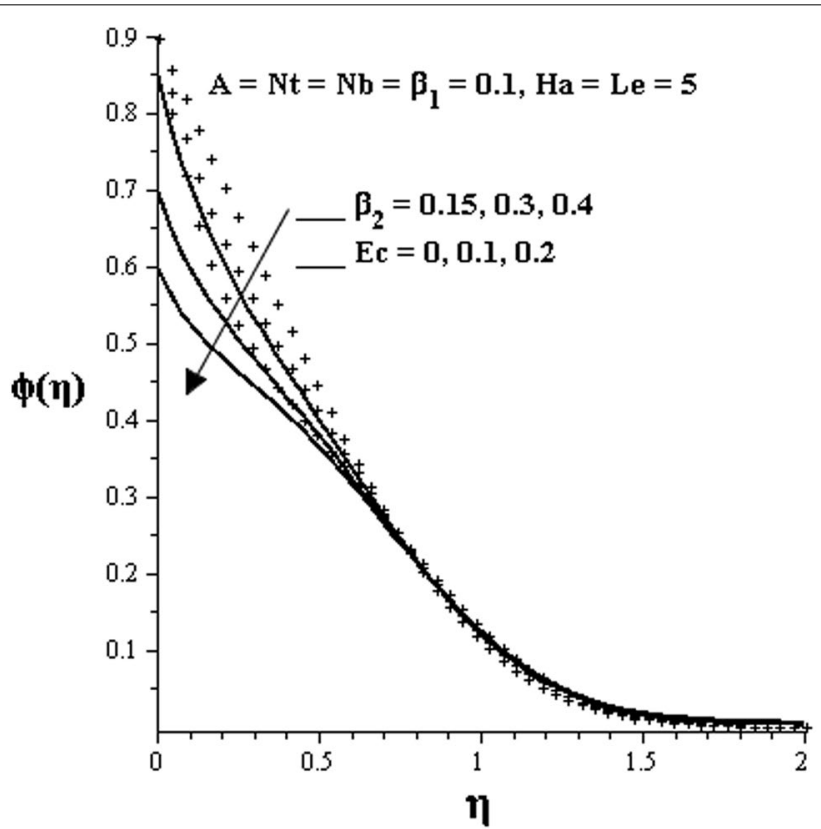

Fig. 9 Effects of $\beta_{2}$ and $E c$ on dimensionless nanoparticle concentration

that both the fluid velocity and the hydrodynamic boundary layer thickness decrease as the unsteadiness parameter $(A)$ and Hartmann number $(H a)$ increases. It is well known that application of a transverse magnetic field orthogonal to the flow direction has a tendency to create a drag force known as Lorentz force which tends to resist the fluid flow and thus reducing its velocity. 


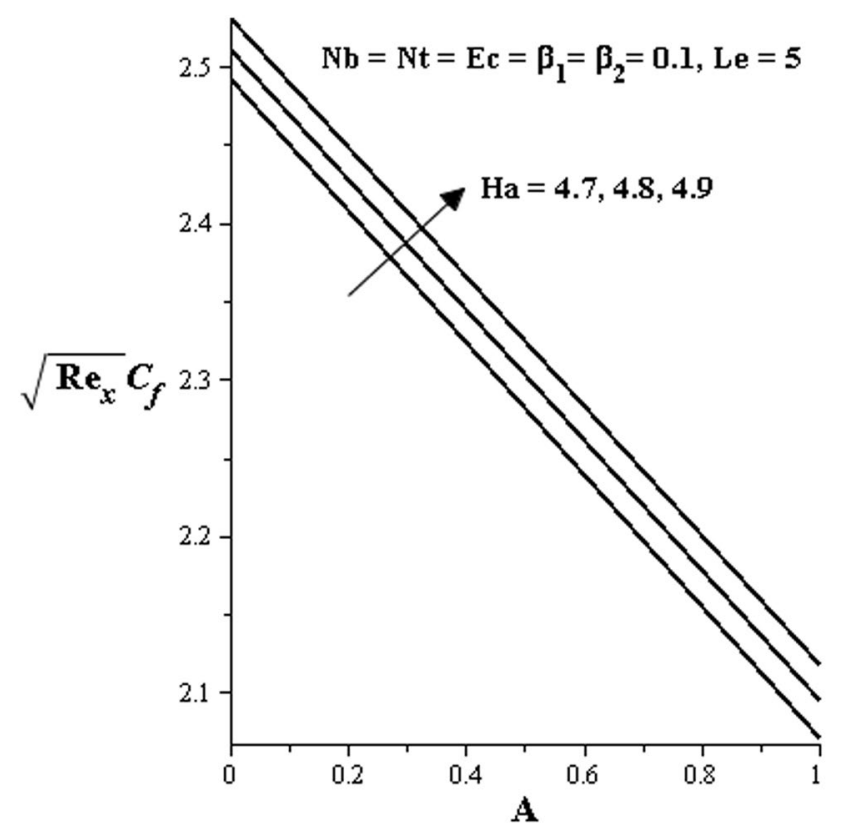

Fig. 10 Effects of $\mathrm{Ha}$ and $\mathrm{A}$ on local skin friction

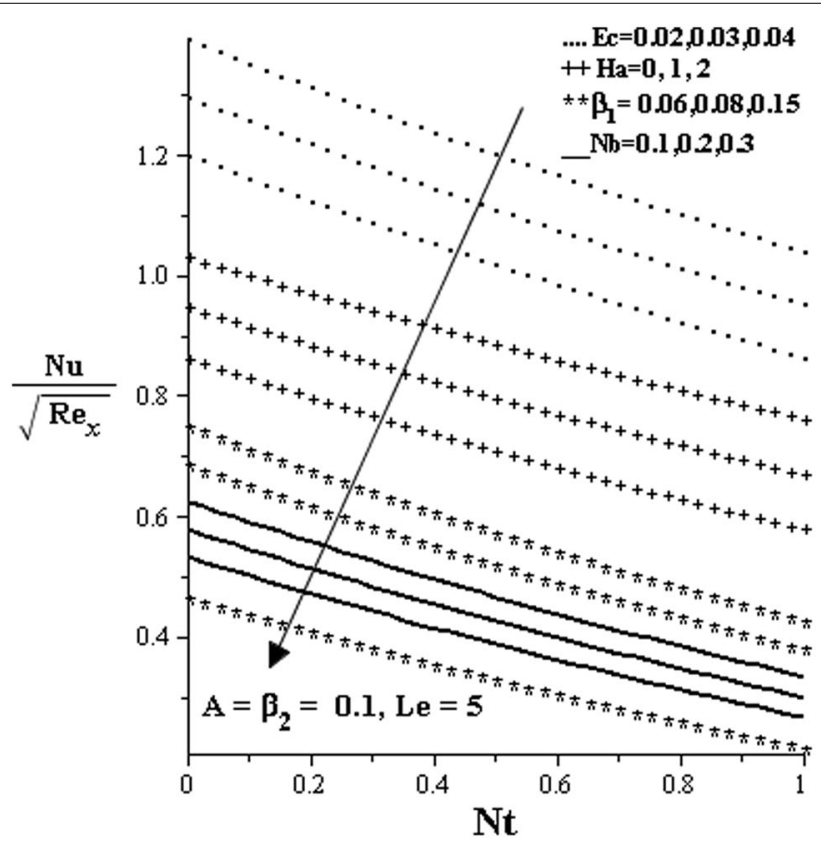

Fig. 11 Effects of $E c, H a, \beta_{1}, \mathrm{Nb}$, and $\mathrm{Nt}$ on local Nusselt number

\section{Temperature profiles}

Figures 3, 4, 5, 6 show the effects of various parameters on the temperature profile. Generally, the temperature is the maximum at the plate surface but decreases exponentially to zero far away from the plate surface satisfying the free stream conditions. As shown in 


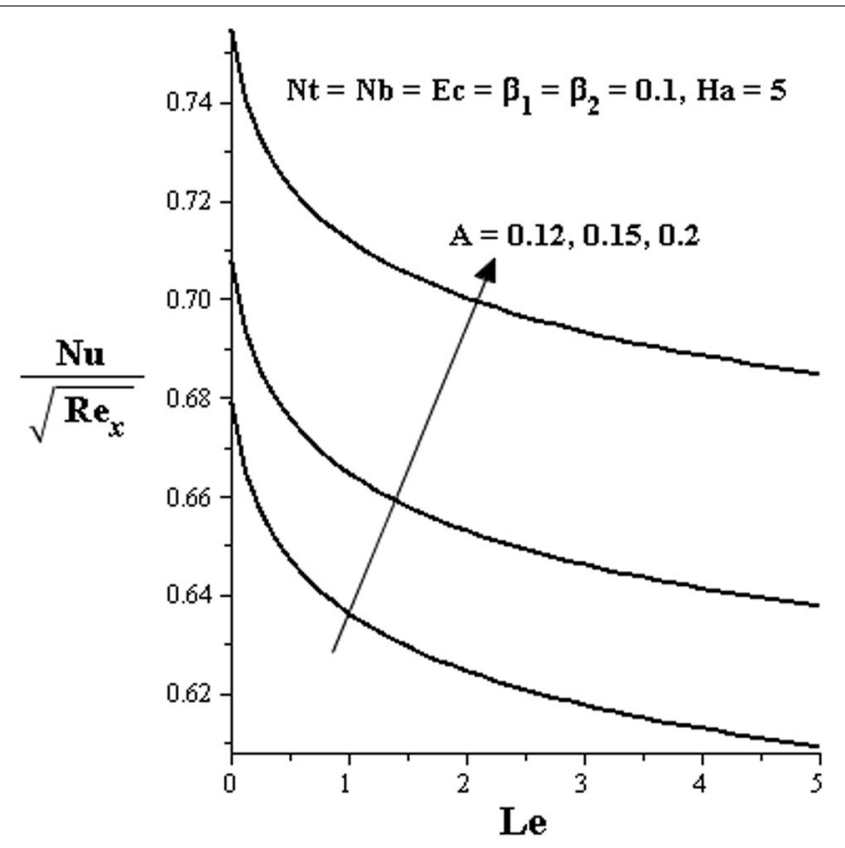

Fig. 12 Effects of $L e$ and $A$ of local Nusselt number

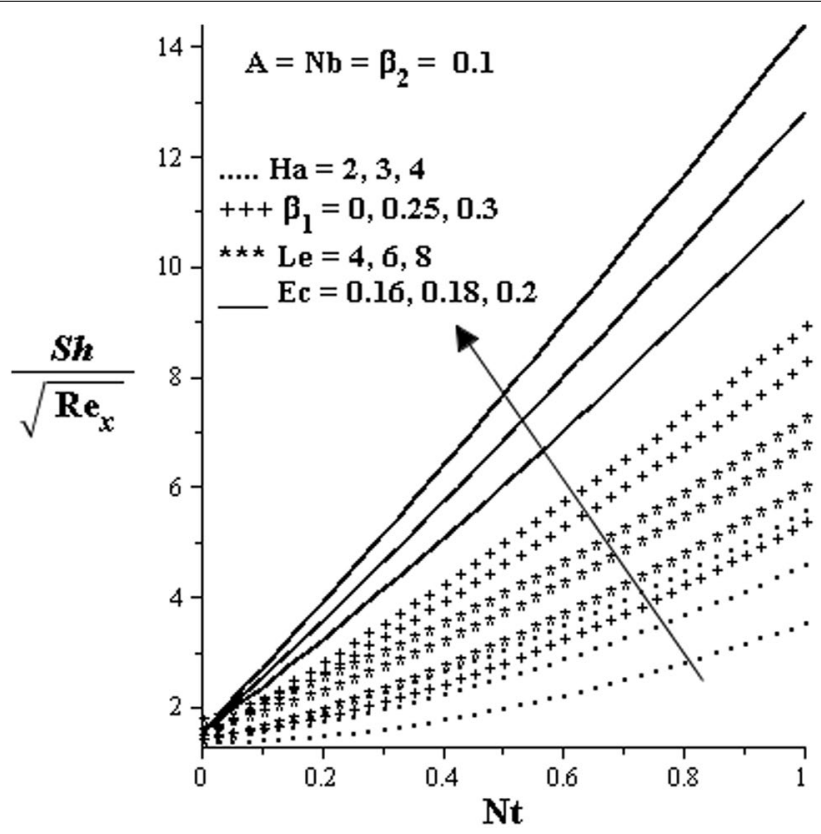

Fig. 13 Effects of $\mathrm{Ha}, E c, \beta_{1}, \mathrm{Nt}$, and Le of local Sherwood number

Fig. 3, an increase in Brownian parameter $N_{\mathrm{b}}$, thermophoresis parameter $N_{\mathrm{t}}$, and Eckert number $E c$ leads to an increase in both the fluid temperature and the thermal boundary layer thickness. This can be attributed to the additional heating due to viscous dissipation, friction, and rapid collision of the nanoparticles as a result of Brownian motion and thermophoresis. Figure 4 shows that increasing Hartmann number $\mathrm{Ha}$ initially increases 


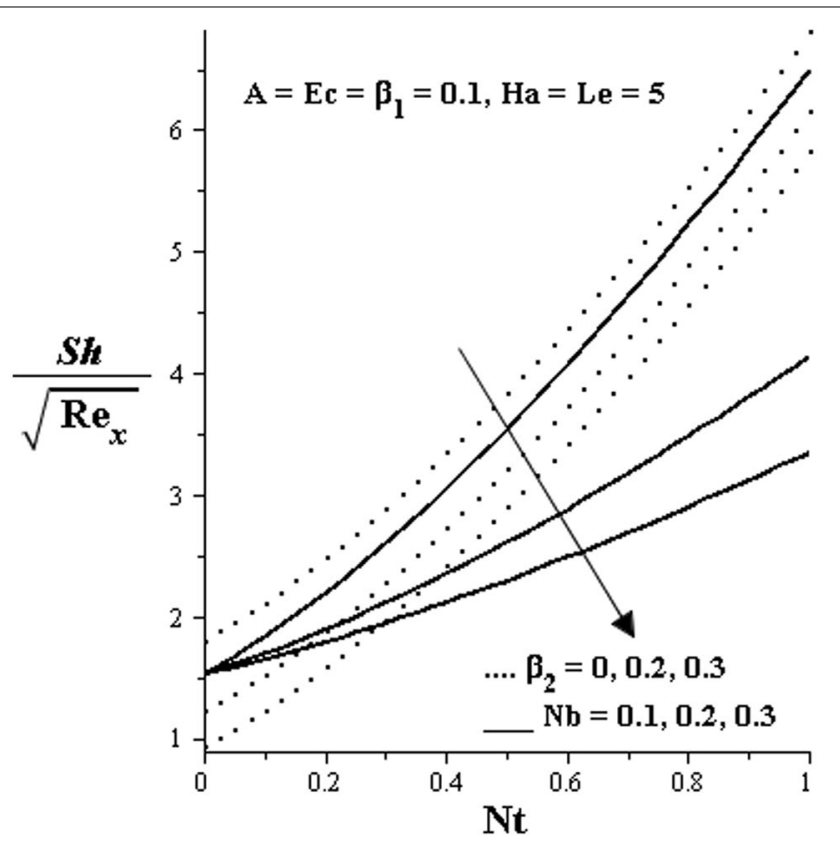

Fig. 14 Effects of $\beta_{2}$ and $\mathrm{Nb}$ of local Sherwood number

Table 1 Computations showing comparison with Aman et al. [20] for different values of $\mathrm{Pr}$ when $A=\beta_{1}=\beta_{2}=H a=\varphi=0$

\begin{tabular}{|c|c|c|c|c|}
\hline$\overline{P r}$ & $\begin{array}{l}f^{\prime \prime}(0) \\
\text { Amani et al. [20] }\end{array}$ & $\begin{array}{l}-\theta^{\prime}(0) \\
\text { Amani et al. }[20]\end{array}$ & $\begin{array}{l}f^{\prime \prime}(0) \\
\text { present }\end{array}$ & $\begin{array}{l}-\theta^{\prime}(0) \\
\text { present }\end{array}$ \\
\hline 0.1 & 2.50997 & 0.26815 & 2.50997 & 0.26815 \\
\hline 0.3 & 2.50997 & 0.40338 & 2.50997 & 0.40338 \\
\hline 0.7 & 2.50997 & 0.52965 & 2.50997 & 0.52965 \\
\hline 6.2 & 2.50997 & 0.59014 & 2.50997 & 0.59014 \\
\hline
\end{tabular}

the fluid temperature at the plate surface, but as the fluid moves from the plate surface the temperature decreases. The increase in temperature is due to ohmic heating as a result of the magnetic effect. On the other hand, as illustrated in Figures 5 and 6, there is a decrease in both the temperature and the thermal boundary layer thickness with an increase in thermal stratification parameter $\beta_{1}$ and unsteadiness parameter $A$. When the thermal stratification effect is taken into consideration, the effective temperature difference between the plate and the ambient fluid decreases; therefore, the temperature is reduced.

\section{Nanoparticle concentration profiles}

Figures 7, 8, 9 show the effects of various parameters on the nanoparticle concentration profile. The nanoparticle concentration is the highest at the plate surface but decreases exponentially to zero free stream value satisfying the boundary conditions. Unlike in the case of temperature, a decrease in the nanoparticle concentration is noted with an increase in unsteadiness parameter $A$, Lewis number $L e$, Brownian parameter $N_{\mathrm{b}}$, 
thermophoresis parameter $N_{\mathrm{t}}$, Hartmann number $H a$, Eckert number $E c$, and solutal stratification parameter $\beta_{2}$.

\section{Effects of parameter variations on $\mathrm{C}_{\mathrm{f}}, \mathrm{Nu}$, Sh}

Figures 10, 11, 12, 13, 14 depict the various pertinent parameters at the plate surface for the skin friction coefficient, the local Nusselt number (rate of heat transfer), and the local Sherwood number with variation in the values of thermophysical parameters embedded in the problem. From Fig. 10, it can be seen that increasing both the unsteadiness parameter $A$ and Hartmann number $H a$ leads to an increase in the skin friction coefficient. This is as expected since both parameters causes an overshoot of the fluid towards the plate surface. From Fig. 11, it is noted increasing the Lewis number $L e$, Brownian parameter $N_{\mathrm{b}}$, thermophoresis parameter $N_{\mathrm{t}}$, Hartmann number $H a$, Eckert number $E c$, and thermal stratification parameter $\beta_{1}$ leads to a decrease in the local Nusselt number which implies that there is reduced heat transfer rate at the plate surface. As seen earlier, the same parameters led to an increase to the fluid temperature; thus, it is expected that with increased temperature within the fluid the rate of heat transfer from the plate to the fluid will be reduced. On the other hand, an increase in the unsteadiness parameter $A$ increases the local Nusselt number, as observed in Fig. 12. It is noted in Figs. 13 and 14 that increasing the Hartmann number $H a$, Eckert number $E c$, Lewis number $L e$, thermophoresis parameter $N_{\mathrm{t}}$, and thermal stratification parameter $\beta_{1}$ increases the Sherwood number, while a decrease in the local Sherwood number is realized by increasing the Brownian parameter $N_{\mathrm{b}}$ and solutal stratification parameter $\beta_{2}$. It well known that at high Lewis number the nanoparticle concentration is low, since the concentration at the plate surface is higher than in the fluid, mass transfer from the plate to the fluid then occurs.

\section{Conclusions}

The combined effects of thermal stratification, solutal stratification, Brownian motion, and thermophoresis on an unsteady hydromagnetic boundary layer flow of a nanofluid over a flat surface are numerically investigated. Using a similarity variable, the governing nonlinear partial differential equations were obtained and transformed to a set of nonlinear differential equations. Numerical results are obtained, then presented graphically for the velocity, temperature, and nanoparticle concentration profiles as well as skin friction coefficient, reduced Nusselt number, and reduced Sherwood number. Our results reveal, among other conclusions, the following:

- The fluid overshoots towards the plate surface with increasing $A$ and $H a$, thus reducing both the fluid velocity and the hydrodynamic boundary layer thickness.

- An increase in $E c, N t, N b$, and $H a$ yields an increase in both the thermal boundary layer thicknesses and temperature, whereas the reverse is noted with increases in $A$ and $\beta_{1}$.

- A decrease in the nanoparticle concentration is noted with increase in $A, L e, N b, N t$, $H a, E c$, and $\beta_{2}$.

- Increasing $A$ and $H a$ increase the skin friction. 
- A decrease in Nusselt number is realized with increased $E c, H a, N b$, and $\beta_{1}$, whereas it increases with increasing values of $A$.

- The local Sherwood number increases with increase in $H a, \beta_{1}, L e, N t$, and $E c$ but reduces with increased $\beta_{2}$ and $N b$.

\section{Abbreviations}

$(u, v)$ : velocity components in $x$ - and $y$-direction; $A$ : unsteadiness parameter $(\lambda / a)$; $B_{0}$ : magnetic field strength $(\mathrm{T})$; $C$ : nanoparticle volume fraction; $C_{\mathrm{f}}$ : skin friction coefficient $\left(\tau_{w} / U_{\infty}^{2} \rho\right) ; C_{0, \infty}$ : constant free stream volume fraction; $D_{\mathrm{B}}$ : Brownian diffusion coefficient; $D_{\mathrm{T}}$ : thermophoresis diffusion coefficient; $E c$ : Eckert number $\left(U_{\infty}^{2} / C_{\mathrm{p}}\left(T_{\mathrm{w}}-T_{0, \infty}\right)\right)$; $H a$ : magnetic parameter $\left(\sigma B_{0}^{2} B_{0}^{2} / \alpha \rho\right)$; $k$ : thermal conductivity (W/mK); Le: Lewis number $\left(v / D_{\mathrm{m}}\right) ; N_{\mathrm{b}}$ : Brownian motion parameter $\left(\tau D_{\mathrm{B}}\left(C_{\mathrm{w}}-C_{0, \infty}\right) / v\right) ; N_{\mathrm{t}}$ : thermophoresis parameter $\left(\tau D_{\mathrm{T}}\left(T_{\mathrm{w}}-T_{0, \infty}\right) / T_{0, \infty} v\right) ; \operatorname{Pr}$ : Prandtl number $(v / \alpha) ; N u$ : Nusselt number $\left(x q_{\mathrm{w}} /\right.$ $\left.k\left(T_{\mathrm{w}}-T_{0, \infty}\right)\right)$; Sh: Sherwood number $\left(x q_{\mathrm{m}} / D_{\mathrm{B}}\left(C_{\mathrm{w}}-C_{0, \infty}\right)\right) ; T$ : temperature of fluid (K)

\section{Greek symbols}

$\psi$ : stream function; $\eta$ : similarity variable; $\beta_{1}$ : thermal stratification parameter; $\beta_{2}$ : solutal stratification parameter; $v$ : kinematic viscosity of fluid $(\mathrm{kg} / \mathrm{m} \mathrm{s}) ; \rho$ : density of fluid $(\mathrm{kg} /$ $\left.m^{3}\right) ; \phi$ : dimensionless concentration function; $\alpha$ : thermal diffusivity $(W / m k)$; $\sigma$ : electrical conductivity of fluid $(S / m) ; \theta$ : dimensionless temperature; $\left(c_{p}\right)_{\mathrm{f}}$ : heat capacity of fluid $(\mathrm{J} /$ $k g K) ;\left(c_{\mathrm{p}}\right)_{\mathrm{p}}$ : heat capacity of nanofluid; $\tau$ : parameter defined by $(\rho C)_{\mathrm{p}} /(\rho C)_{\mathrm{f}} ; \mu$ : dynamic viscosity of fluid $(\mathrm{kg} / \mathrm{ms})$

\section{Subscripts}

$\infty$ : condition at the free stream; w: condition at the surface

\section{Authors' contributions}

Both authors were actively involved in the formulation of the problem, literature review, and simulations. Both authors read and approved the final manuscript.

\section{Author details}

${ }^{1}$ Department of Mathematics, Kenyatta University, Nairobi, Kenya. ${ }^{2}$ Faculty of Military Science, Stellenbosch University, Cape Town, South Africa.

\section{Acknowledgements}

None.

Competing interests

The authors declare that they have no competing interests.

Funding

There is no funding towards the publication of this manuscript.

Ethics approval and consent to participate

This study does not involve human participants, human data, or human tissue.

\section{Publisher's Note}

Springer Nature remains neutral with regard to jurisdictional claims in published maps and institutional affiliations.

Received: 27 March 2017 Accepted: 3 May 2017

Published online: 12 May 2017

\section{References}

1. Makinde OD (2011) On MHD mixed convection with Soret and Dufour effects past a vertical plate embedded in a porous medium. LAAR 41(1):63-68 
2. Saha LK, Siddiqa S, Hossain MA (2011) Effect of hall current on MHD natural convection flow from vertical permeable flat plate with uniform surface heat flux. Appl Math Mech Engl 3(9):1127-1146

3. Rout BR, Panda SK, Panda S (2013) MHD heat and mass transfer of chemical reaction fluid flow over a moving vertical plate in presence of heat source with convective surface boundary condition. Int J Chem Eng. doi:10.1155/2013/296834

4. Socolofsky AS, Jirka GH (2004) Stratified flow and buoyant mixing. Eng Lect Notes. Texas A\&M University, College Station

5. Singh G, Sharma PR, Chamkha AJ (2010) Effect of thermally stratified ambient fluid on MHD convective flow along a moving non-isothermal vertical plate. Int J Phys Sci 5(3):208-215

6. Saha SC, Hossain MA (2004) Natural convection flow with combined buoyancy effects due to thermal and mass diffusions in a thermally stratified media. NonLinear Anal 9(1):89-102

7. Mukhopadhyay S, Mondal C, Gorla SR (2012) Effects of thermal stratification on flow and heat transfer past a porous vertical stretching surface. Heat Mass Transf 48:915-921

8. Choi SUS (1995) Enhancing thermal conductivity of fluids with nanoparticle. In: Siginer DA, Wang HP (eds) Development and applications of non-Newtonian flow. The American Society of Mechanical Engineers, New York, pp 227-233

9. Srinivasacharya D, Upendar M (2013) Effect of double stratification on MHD free convection in a micropolar fluid. J Egypt Math Soc. doi:10.1016/j.joems.2013.02.006

10. Narayana L, Murthy PA (2006) Free convective heat and mass transfer in a doubly stratified non-Darcy porous medium. J Heat Transf 128:1204-1212

11. Ibrahim W, Shankar B (2013) MHD boundary layer flow and heat transfer of a nanofluid past a permeable stretching sheet with velocity, thermal and solutal slip boundary conditions. J Comput Fluids 75:1-10

12. Makinde OD, Olanrewaju PO, Charles WM (2011) Unsteady convection with chemical reaction and radiative heat transfer past a flat porous plate moving through a binary mixture. Afr Mat 22(2011):65-78

13. Chamkha AJ, Rashady AM, Al-Meshaieiz E (2011) Melting effect on unsteady hydromagnetic flow of a nanofluid past a stretching sheet. Int J Chem Reactor Eng 9(113):1

14. Makinde OD (2012) Computational modelling of MHD unsteady flow and heat transfer towards a flat plate with Navier slip and Newtonian heating. Braz J Chem Eng 29(1):159-166

15. Khan MS, Karim I, Ali LE, Islam A (2012) Unsteady MHD free convection boundary layer flow of a nanofluid along a stretching sheet with thermal radiation and viscous dissipation effects. Int Nano Lett 2:24

16. Vajravelu K, Prasad KV, Ng C (2013) Unsteady convective boundary layer flow of a viscous fluid at a vertical surface with variable fluid properties. Nonlinear Anal Real 14:455-464

17. Makinde OD, Chinyoka T (2013) Numerical investigation of buoyancy effects on hydromagnetic unsteady flow through a porous channel with suction/injection. J Mech Sci Tech 27(5):1557-1568

18. Olanrewaju AM, Makinde OD (2013) On boundary layer stagnation point flow of a nanofluid over a permeable flat surface with Newtonian heating. Chem Eng Commun 200:836-852

19. Ibrahim W, Shanker B (2012) Unsteady MHD boundary layer flow and heat transfer due to stretching sheet in the presence of heat source or sink by quasi linearization technique. Int J Appl Math Mech 8(7):18-30

20. Aman F, Ishak A, Pop I (2011) Mixed convection boundary layer flow near stagnation point on vertical surface with slip. Appl Math Mech 32(12):1599-1606

\section{Submit your next manuscript to BioMed Central and take full advantage of:}

- Convenient online submission

- Thorough peer review

- No space constraints or color figure charges

- Immediate publication on acceptance

- Inclusion in PubMed, CAS, Scopus and Google Scholar

- Research which is freely available for redistribution

Submit your manuscript at www.biomedcentral.com/submit 